\title{
Estimating the age of Heliconius butterflies from calibrated photographs
}

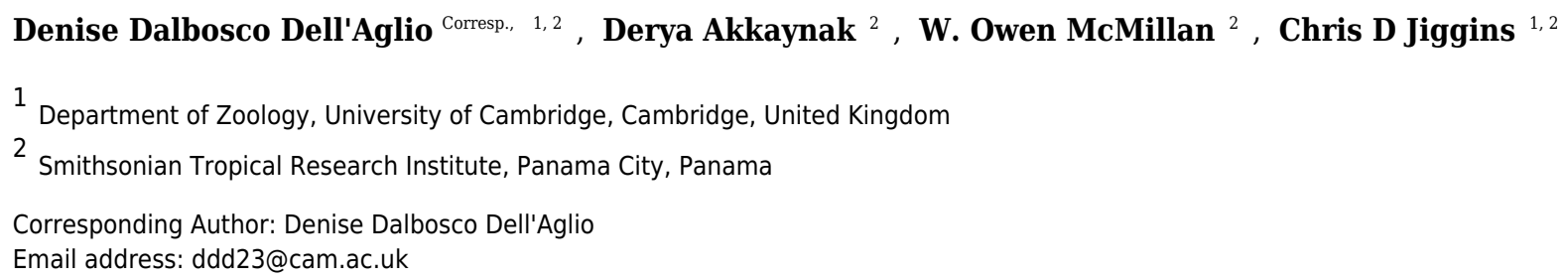

Mating behaviour and predation avoidance in Heliconius involve visual colour signals, however there is considerable inter-individual phenotypic variation in the appearance of colours. In particular, the red pigment varies from bright crimson to faded red. It has been thought that this variation is primarily due to pigment fading with age, although this has not been explicitly tested. Previous studies have shown the importance of red patterns in mate choice and that birds and butterflies might perceive these small colour differences. Using digital photography and calibrated colour images, we investigated whether the hue variation in the forewing dorsal red band of Heliconius melpomene rosina corresponds with age. We found that the red hue and age were highly associated, suggesting that red colour can indeed be used as a proxy for age in the study of wild-caught butterflies. 
1 Estimating the age of Heliconius butterflies from calibrated photographs

2

3 Denise Dalbosco Dell'Aglio 1,2 *

4 Derya Akkaynak ${ }^{2}$

5 W. Owen McMillan ${ }^{2}$

6 Chris D. Jiggins ${ }^{1,2}$

7

$8 \quad{ }^{1}$ Butterfly Genetics Group, Department of Zoology, University of Cambridge, UK

$9 \quad{ }^{2}$ Smithsonian Tropical Research Institute, Panama City, Panama

10

11 *Corresponding author:

12 Department of Zoology, University of Cambridge,

13 Downing St, Cambridge, CB2 3EJ, UK

14 e-mail: ddd23@cam.ac.uk

15

16

17

18

19

20

21

22

23 Abstract 
24 Mating behaviour and predation avoidance in Heliconius involve visual colour signals, however 25 there is considerable inter-individual phenotypic variation in the appearance of colours. In 26 particular, the red pigment varies from bright crimson to faded red. It has been thought that this 27 variation is primarily due to pigment fading with age, although this has not been explicitly tested.

28 Previous studies have shown the importance of red patterns in mate choice and that birds and 29 butterflies might perceive these small colour differences. Using digital photography and 30 calibrated colour images, we investigated whether the hue variation in the forewing dorsal red 31 band of Heliconius melpomene rosina corresponds with age. We found that the red hue and age 32 were highly associated, suggesting that red colour can indeed be used as a proxy for age in the 33 study of wild-caught butterflies. 
47 Butterflies are some of the most colourful living animals and their bright wing colours have attracted the attention of scientists and artists alike. Multiple selective factors affect the evolution of butterfly wing colours, which might be tuned to the visual systems of potential mates and

50 predators. This might be particularly true for the genus Heliconius, which exhibits conspicuous 51 colours as a warning signal of toxicity (Benson, 1972; Langham, 2004), and find and choose 52 mates based on these same colour signals that are involved in predator avoidance (Jiggins et al., 53 2001; Estrada \& Jiggins, 2008).

Although mating behaviour and predation avoidance in Heliconius is highly linked to colour, previous research has shown that some Heliconius species exhibit considerable phenotypic variation in colour. Analysis of the colour patterns of two polymorphic mimic butterflies, Heliconius numata and the genus Melinaea, suggests that small differences in contrast can be perceived by butterflies and birds (Llaurens, Joron \& Théry, 2014). Moreover, variation of wing colour spectra between populations of Heliconius timareta indicates that their colours are locally adapted for mimicry in very precise ways (Mérot et al., 2016).

Most studies of colouration in Heliconius butterflies have focused on the genetic basis for colour variation (Reed, Mcmillan \& Nagy, 2008; Reed et al., 2011; Pardo-Diaz et al., 2012). Convergent gene expression in $H$. melpomene and $H$. erato is associated with red wing elements (Pardo-Diaz \& Jiggins, 2014). Moreover, the H. melpomene gene responsible for red colour pattern is genetically linked to a preference for red (Naisbit, Jiggins \& Mallet, 2001; Merrill et al., 2011). From a morphological point of view, wing scales have ultra-structural differences which are correlated with pigmentation (Gilbert et al., 1988; Aymone, Valente \& de Araújo, 2013). Red/brown scales in Heliconius are pigmented with xanthommatin and dihydroxanthommatin and vary in colour from bright red to brown due to variations in the 
70 pigment oxidation state (Gilbert et al., 1988). However, there is also phenotypic variation in red

71 among individuals that do not differ genetically, in wing regions with the same pigmentation and

72 ultrastructure, with variation in colour from bright crimson to faded red.

73 It has been suggested that this variation in red is due to oxidation of the red 74 dihydroxanthommatin pigment as individuals age (Crane, 1954; Ehrlich \& Gilbert, 1973; Gilbert 75 et al., 1988). Previous studies have taken advantage of this phenomenon to measure arbitrary age 76 structure in Heliconius using wing condition such as wear, dull colours and scale loss (Ehrlich \& 77 Gilbert, 1973; Walters et al., 2012). We here investigated whether red colour can be used as a 78 proxy for age in the study butterflies removing human vision bias. We used analysis based on digital photography to investigate the association between wing colouration and age, a methodology increasingly common in studies of animal coloration due to its high-end technology

81 and affordability (Stevens et al., 2007; Akkaynak et al., 2014). Here, we created an unbiased 82 methodology to quantify age based on the "redness" of the forewing red band in Heliconius 83 butterflies.

\section{Material and Methods}

86 To quantify changes in the dorsal forewing red band, we used a set of 55 Heliconius melpomene 87 rosina Boisduval 1870 wings from Owen McMillan's collection in Gamboa, Panama, raised in 88 insectaries and of known age (in days after emergence).

89 To objectively characterise colour, photographs of the wings were taken using an 90 Olympus OM-D EM-1 digital camera with an Olympus Zuiko Digital ED $60 \mathrm{~mm} \mathrm{f} / 2.8$ macro

91 lens (Olympus, Center Valley, PA, USA). Forewing specimens were photographed against a 92 Kodak R-27 Gray card, with Munsell 18\% reflectance (Eastman Kodak, Rochester, NY, USA). 
93 Camera RAW images were converted to DNG format using the Adobe DNG Converter®, and

94 white balanced and colour corrected according to equations 5-8 described in (Akkaynak et al., 95 2014) using an Xrite Color Checker (Xrite, Grand Rapids, MI, USA). For illumination, a Bolt 96 VM-110 LED macro ring light was used (Gradus Group LLC, New York, NY, USA). Image 97 manipulations were done using custom scripts written in MATLAB ${ }^{\circledR}$ in wide gamut Kodak 98 ProPhoto RGB colour space.

99 Following colour calibration, RGB images were projected to $\mathrm{L}^{*} \mathrm{a} * \mathrm{~b} *$ colour space 100 (Wyszecki \& Stiles, 1982) and the $\mathrm{a}^{*}$ channel of each image was given a threshold at a*> 10 to 101 segment the red patch automatically and obtain a binary mask. In the $\mathrm{L}^{*} \mathrm{a}^{*} \mathrm{~b} *$ colour space, the $102 \mathrm{a}^{*}$ channel takes on values between -128 and +128 , and represents red-green opponency. The $\mathrm{b}^{*}$ 103 channel also takes on values between -128 and +128 , and represents yellow-green opponency. 104 The $\mathrm{L}^{*}$ channel varies between 0 and 100, and is a measure of lightness, with 100 being a bright 105 white. Thus in this colour space, the more positive the a* value, the "redder" an image appears. 106 The resulting binary mask was cleaned of isolated pixels using first morphological area opening, 107 then closing. The automatically made masks were checked visually to ensure no pixels outside 108 the red patch area or severe scale loss patches were included. The $\mathrm{a}^{*}$ and $\mathrm{b}^{*}$ values of the pixels 109 inside the red patch area were averaged to obtain a representative colour for each specimen.

110 First, to test for differences between sexes, a t-test was performed. Next, to test for 111 association between age and colour, a linear regression analysis was performed between age 112 (days after emergence) and red measurement $(* a)$. In addition, wings were sorted into groups 113 using age/colour categories based on previous work in H. ethilla (Ehrlich \& Gilbert, 1973).

114 These subjective categories were named based on the appearance of the red band to human 115 vision: crimson (fresh wings), red (intermediate) and faded red (worn) (Figure 1). The 
116 categorical data was used to investigate the efficiency of human vision in categorizing subjective

117 wing colouration.

118

119

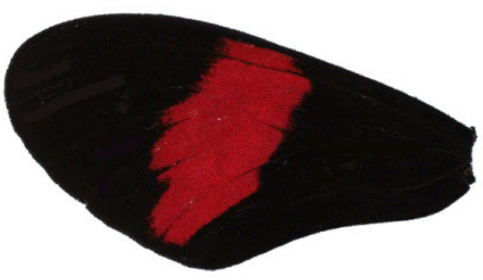

crimson

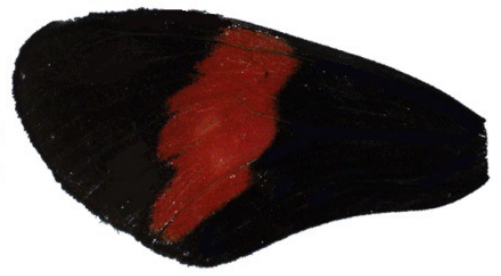

red

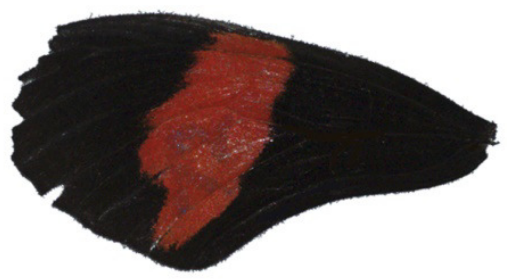

faded red

120

Figure 1. Forewings of Heliconius melpomene rosina. Categories based on the appearance of colours to human vision: crimson (fresh wings), red (intermediate) and faded red (worn).

Results and Discussion

125 Through calibrated digital images, we quantified the redness of Heliconius wings and found a

126 strong association between age and fading of the colour (Figure 2). We analysed 37 females and

12718 males and found no significant difference in colour between the sexes $\left(\mathrm{t}_{53,55}=0.409, P=\right.$ 128 0.684), so both were combined in subsequent analyses. Our results showed that redness and age 129 were highly associated $\left(\mathrm{t}_{53,55}=-7.461, P<0.001\right)$, indicating that younger individuals have 130 quantifiably "redder" patches. This suggests that the forewing dorsal red band changes colour

131 with age as had been suggested previously (Figure 2).

132 


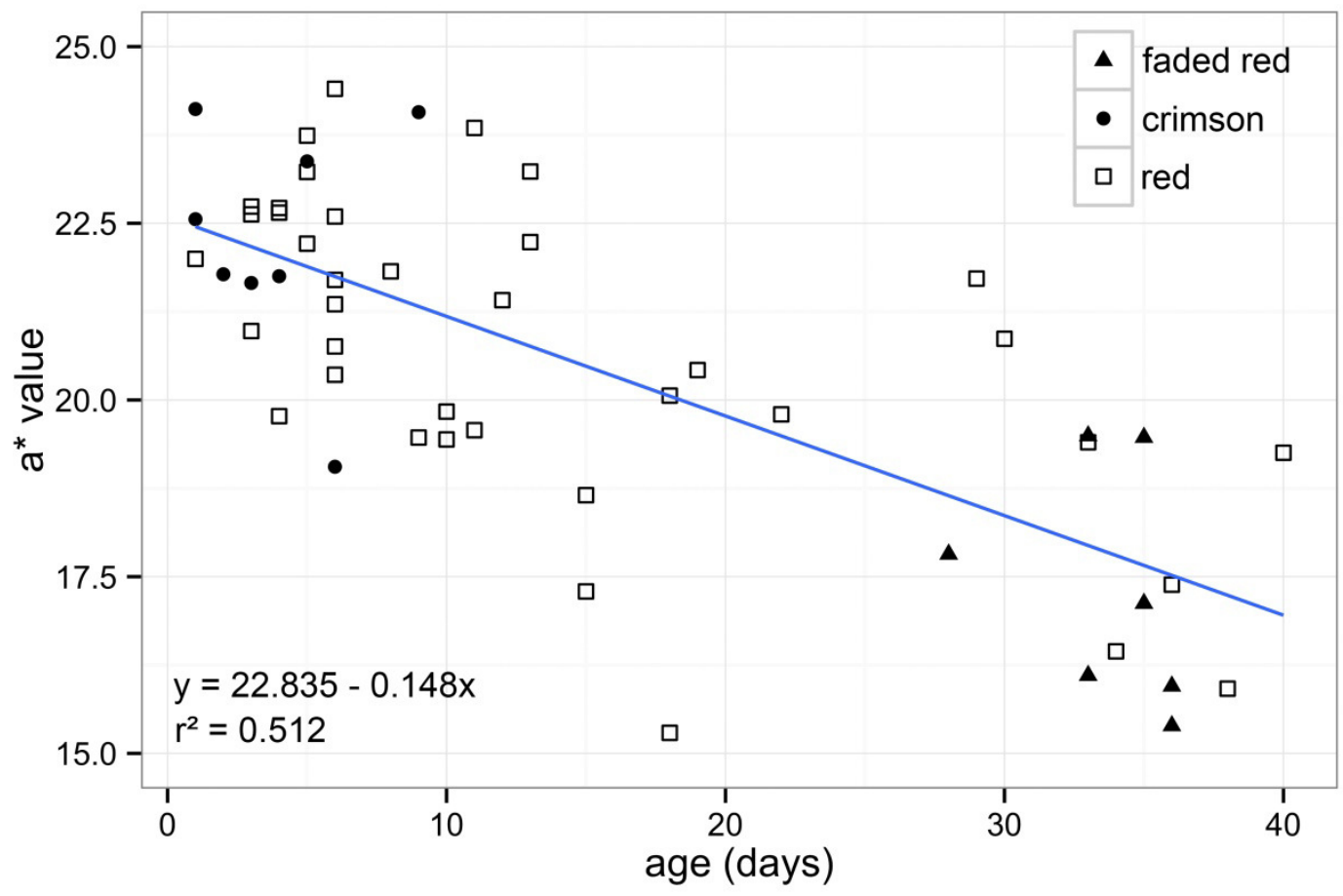

133

134 Figure 2. Forewing red band changes colour with age. Association between redness (a* value) 135 and age in days after emergence in Heliconius melpomene rosina forewing dorsal red band $\left(\mathrm{t}_{53,55}\right.$ $136=-7.461, P<0.001$ ). Human visual categories: crimson (filled circles, $\mathrm{n}=9$ ), red (open squares, $137 \mathrm{n}=39$ ) and faded red (filled triangles, $\mathrm{n}=7$ ). natural age structure of wild populations (Ehrlich \& Gilbert, 1973). We have also shown that

141 through calibrated photographs it is possible to distinguish more colour categories when 142 compared to the limitations of the human vision. Although human visual categories do not fit 143 especially well with the correlation (Figure 2), the "crimson" category included all specimens 144 that were less than 10 days old and "faded red" included specimens that were all older than 25 145 days (Figure 2). Also, "faded red" might contain information about scale loss, in which was not 146 explored in our methodology. 
148 Halyomorpha brevis, with the change in red stronger in males (Niva \& Takeda, 2002). Similar

149 colour fading was found as a consequence of direct sun exposure in bees, Bombus huntii, which

150 colour hue was correlated with wing wear and therefore could be a reliable measure of age (Koch

151 et al., 2014). In butterfly wings, age-based colour fading has also been shown in Colias

152 eurytheme, in which fading of structural colour was the most accurate predictor of male age.

153 Females of this species choose their partners based on age, since new males produce more

154 nutritious spermatophores such that colour might be a useful cue for mate choice (Kemp, 2006).

155 It is less clear whether there would be a similar benefit to such age discrimination in

156 Heliconius. Females mate only once or a few times in their lifetime, depending on species, and

157 the first mating occurs soon after eclosion (Walters et al., 2012). In contrast, males can mate

158 throughout their life and there is no evidence that spermatophore quality is influenced by male

159 age, although this has not been directly tested. Male choosiness in Heliconius is well documented

160 (Jiggins, Estrada \& Rodrigues, 2004; Estrada \& Jiggins, 2008) as the spermatophore represents a

161 considerable nutrient investment, providing the female with amino acids used in egg production

162 (Boggs, 1981). Females are also likely to be choosy but this has been less well documented. Age,

163 perhaps signalled by colour cues, might be a cue for mate choice in Heliconius and this would be

164 interesting to test explicitly.

165 Furthermore, $H$. erato has red lateral filtering pigments which shift red receptor

166 sensitivity, allowing butterflies to distinguish colours in the red-green spectrum with just a single

167 LW-sensitive opsin (Zaccardi et al., 2006; McCulloch, Osorio \& Briscoe, 2016). This means that

168 Heliconius likely have better abilities to distinguish slight differences in the red colour range as

169 compared to other nymphalids (Zaccardi et al., 2006). It would therefore be interesting to test 
170 whether adults can distinguish colours across the range demonstrated here among individuals of

171 different ages. If age were an important trait in sexual selection, perhaps red filtering pigments

172 are in part an adaptation for better mate discrimination in this range. It would be interesting to

173 investigate how these colour differences would be seen through Heliconius vision.

174 It is also interesting to speculate about whether the fading of pigments might influence

175 how predators perceive these butterflies. Although some degree of predator generalization is

176 likely, it is also known that predators can distinguish fairly subtle differences in hue (Langham,

177 2004). Further experiments would be needed to determine whether colour fading might incur

178 some cost in terms of increased predation. In conclusion, we have demonstrated how colour

179 could be used to estimate age in population structure studies, and provided the groundwork for

180 future studies of the fitness consequences of fading colours in Heliconius for mate choice and 181 mimicry.

182

183 Acknowledgements

184 We are very grateful to Adriana Tapia for her help with the butterfly collection, the Smithsonian

185 Tropical Research Institute for logistical assistance, and three anonymous reviewers for valuable 186 comments on the manuscript.

\section{References}

190 Akkaynak D., Treibitz T., Xiao B., Gurkan UA., Allen JJ., Demirci U., Hanlon RT. 2014. Use of commercial off-the-shelf digital cameras for scientific data acquisition and scene-specific color calibration. Journal of the Optical Society of America 31:312-321. 
193 Aymone ACB., Valente VLS., de Araújo AM. 2013. Ultrastructure and morphogenesis of the

194 wing scales in Heliconius erato phyllis (Lepidoptera: Nymphalidae): What

195 silvery/brownish surfaces can tell us about the development of color patterning?

196 Arthropod Structure \& Development 42:349-359. DOI: 10.1016/j.asd.2013.06.001.

197 Benson WW. 1972. Natural Selection for Mullerian Mimicry in Heliconius erato in Costa Rica.

$198 \quad$ Science 176:936-939.

199 Boggs CL. 1981. Selection Pressures Affecting Male Nutrient Investment at Mating in

$200 \quad$ Heliconiine Butterflies. Evolution 35:931-940.

201 Crane J. 1954. Spectral Reflectance Characteristics of Butterflies (Lepidoptera) from Trinidad, B. W. I. Zoologica 39:85-112.

203 204

Ehrlich PR., Gilbert LE. 1973. Population Structure and Dynamics of the Tropical Butterfly Heliconius ethilla. Biotropica 5:69-82.

Estrada C., Jiggins CD. 2008. Interspecific sexual attraction because of convergence in warning colouration: is there a conflict between natural and sexual selection in mimetic species? Journal of Evolutionary Biology 21:749-760. DOI: 10.1111/j.1420-9101.2008.01517.x.

Gilbert LE., Forrest HS., Schultz TD., Harvey DJ. 1988. Correlations of ultrastructure and pigmentation suggest how genes control development of wing scales of Heliconius butterflies. Journal of Research on the Lepidoptera 26:141-160.

Jiggins CD., Estrada C., Rodrigues A. 2004. Mimicry and the evolution of premating isolation in Heliconius melpomene Linnaeus. Journal of Evolutionary Biology 17:680-691. DOI: 10.1111/j.1420-9101.2004.00675.x.

Jiggins CD., Naisbit RE., Coe RL., Mallet J. 2001. Reproductive isolation caused by colour pattern mimicry. Nature 411:302-305. 
216 Kemp DJ. 2006. Heightened phenotypic variation and age-based fading of ultraviolet butterfly 217 wing coloration. Evolutionary Ecology Research 8:515-527.

218 Koch JB., Love B., Klinger E., Strange JP. 2014. The effect of photobleaching on bee

219 (Hymenoptera: Apoidea) setae color and its implications for studying aging and behavior. $220 \quad$ Journal of Melittology 38:1-10.

221 Langham GM. 2004. Specialized avian predators repeatedly attack novel color morphs of 222 Heliconius butterflies. Evolution 58:2783-2787.

223

224

225

226 227 228 229 230 231

232

233 234 235 236 237 238

Llaurens V., Joron M., Théry M. 2014. Cryptic differences in colour among Müllerian mimics: how can the visual capacities of predators and prey shape the evolution of wing colours? Journal of Evolutionary Biology 27:531-540. DOI: 10.1111/jeb.12317.

McCulloch KJ., Osorio D., Briscoe AD. 2016. Sexual dimorphism in the compound eye of Heliconius erato: a nymphalid butterfly with at least five spectral classes of photoreceptor. Journal of Experimental Biology 219:2377-2387. DOI: 10.1242/jeb.136523.

Mérot C., Le Poul Y., Théry M., Joron M. 2016. Mimicry refinement: Phenotypic variations tracking the local optimum. Journal of Animal Ecology 85:1056-1069. DOI: $10.1111 / 1365-2656.12521$.

Merrill RM., Van Schooten B., Scott JA., Jiggins CD. 2011. Pervasive genetic associations between traits causing reproductive isolation in Heliconius butterflies. Proceedings of the Royal Society B 278:511-518. DOI: 10.1098/rspb.2010.1493.

Naisbit RE., Jiggins CD., Mallet J. 2001. Disruptive sexual selection against hybrids contributes to speciation between Heliconius cydno and Heliconius melpomene. Proceedings of the Royal Society B 268:1849-1854. DOI: 10.1098/rspb.2001.1753. 
239 Niva CC., Takeda M. 2002. Color changes in Halyomorpha brevis (Heteroptera: Pentatomidae) 240 correlated with distribution of pteridines: Regulation by environmental and physiological 241 factors. Comparative Biochemistry and Physiology Part B 132:653-660. DOI: $10.1016 / \mathrm{S} 1096-4959(02) 00081-7$.

243 Pardo-Diaz C., Jiggins CD. 2014. Neighboring genes shaping a single adaptive mimetic trait. Evolution \& Development 16:3-12. DOI: 10.1111/ede.12058.

245

246

247

248

250

251

252

253

254

255

256

257

258

259

260

Pardo-Diaz C., Salazar C., Baxter SW., Merot C., Figueiredo-Ready W., Joron M., McMillan WO., Jiggins CD. 2012. Adaptive introgression across species boundaries in Heliconius butterflies. PLoS genetics 8:e1002752. DOI: 10.1371/journal.pgen.1002752.

Reed RD., Mcmillan WO., Nagy LM. 2008. Gene expression underlying adaptive variation in Heliconius wing patterns: non-modular regulation of overlapping cinnabar and vermilion prepatterns. Proceedings of the Royal Society B 275:37-46. DOI: 10.1098/rspb.2007.1115.

Reed RD., Papa R., Martin A., Hines HM., Counterman BA., Pardo-Diaz C., Jiggins CD., Chamberlain NL., Kronforst MR., Chen R., Halder G., Nijhout HF., McMillan WO. 2011. Optix Drives the Repeated Convergent Evolution of Butterfly Wing Pattern Mimicry. Science 333:1137-41. DOI: 10.1126/science.1208227.

Stevens M., Párraga CA., Cuthill IC., Partridge JC., Troscianko TS. 2007. Using digital photography to study animal coloration. Biological Journal of the Linnean Society 90:211-237.

Walters JR., Stafford C., Hardcastle TJ., Jiggins CD. 2012. Evaluating female remating rates in light of spermatophore degradation in Heliconius butterflies: pupal-mating monandry 
261 versus adult-mating polyandry. Ecological Entomology 37:257-268. DOI:

262 10.1111/j.1365-2311.2012.01360.x.

263 Wyszecki G., Stiles WS. 1982. Color Science: Concepts and Methods, Quantitative Data and $264 \quad$ Formulae. New York: Wiley.

265 Zaccardi G., Kelber A., Sison-mangus MP., Briscoe AD. 2006. Color discrimination in the red 266 range with only one long-wavelength sensitive opsin. Journal of Experimental Biology 267 209:1944-1955. DOI: 10.1242/jeb.02207.

268 